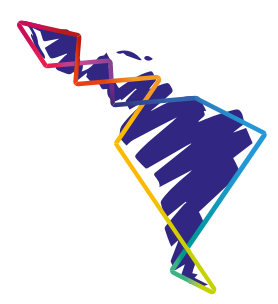

\title{
Educación a distancia: discriminación en el acceso a Internet y la tecnología de estudiantes de I y II ciclos de la educación general básica
}

\author{
Distance education: Discrimination \\ in access to internet and technology \\ of students of I and II Cycles of Basic \\ General Education
}

\section{Educação a distância: Discriminação no acesso à internet e à tecnologia dos alunos do I e II Ciclos do Ensino Básico Geral}

\author{
M. Sc. Viriam M ejías Padilla ${ }^{1}$ \\ M. Sc. Erika Badilla Sánchez²
}

\begin{abstract}
Resumen
El presente artículo se enmarca en el contexto de la educación a distancia, la cual se implementó en Costa Rica en el 2020, para frenar el contagio por Covid-19. El objetivo es problematizar la desigualdad educativa que enfrentan aquellos estudiantes sin acceso a internet y a la tecnología,
\end{abstract}

Recibido: 25-6-2021 - Aceptado: 5-10-2021

1 Doctora en educación con especialidad en mediación pedagógica. Magíster en gerencia de enfermería. Licenciada en enfermería. Directora de enfermería, Hospital San Juan de Dios, Caja Costarricense de Seguro Social, Costa Rica. Directora del Posgrado de Enfermería, Universidad Hispanoamericana, Costa Rica. Imparte los cursos de Gestión de Servicios de Enfermería I y Supervisión y Evaluación del Desempeño. Costarricense. Correo electrónico: vmejias@ccss.sa.cr. (D) https://orcid.org/0000-0001-8778-5450

2 Doctora en educación con especialidad en mediación pedagógica. Magíster en salud mental y enfermería. Licenciada en enfermería. Enfermera especialista en salud mental, Hospital San Juan de Dios, Caja Costarricense de Seguro Social, Costa Rica. Imparte los cursos de Psicología Infantil, Campo Clínico en Salud Mental y Comportamiento Organizacional, en el Programa de Posgrado de Enfermería, Universidad Hispanoamericana, Costa Rica. Costarricense.Correo electrónico: eka.badilla@gmail.com. (D) https://orcid. org/0000-0002-6363-4209 
lo que limita su derecho a la formación. Además, se presenta la importancia de establecer estrategias que permitan incluir, a futuro, los recursos tecnológicos en los procesos educativos, de forma que se pueda brindar una educación de mayor calidad y adecuada al panorama actual. Aunado, se concluye que el acceso a internet y a los recursos tecnológicos es el primer paso para garantizar el derecho a la educación, en la modalidad a distancia.

Palabras clave: educación a distancia, discriminación, acceso, tecnología.

\begin{abstract}
This article is framed in the context of distance education, which was implemented in Costa Rica in 2020 to stop the contagion by Covid-19. The objective is to problematize the educational inequality faced by those students without access to the internet and technology, which limits their right to education. In addition, the importance of establishing strategies that allow future inclusion of technological resources within educational processes is presented, so that a higher quality education can be provided and appropriate to the current context. It is concluded that access to the internet and technological resources are the first step to guarantee the right to education in the distance education modality.
\end{abstract}

Keywords: distance education, discrimination, access, technology.

\title{
Resumo
}

Este artigo se enquadra no contexto da educação a distância, implementada na Costa Rica em 2020 para conter o contágio da Covid-19. O objetivo é problematizar a desigualdade educacional enfrentada por alunos sem acesso à internet e tecnologia, o que limita seu direito à educação. Além disso, apresenta-se a importância de se estabelecer estratégias que possibilitem a inclusão futura de recursos tecnológicos nos processos educacionais, para que uma educação de maior qualidade e adequada ao contexto atual. Além disso, conclui-se que o acesso à internet e os recursos tecnológicos são o primeiro passo para garantir o direito à educação na modalidade de educação a distância.

Palavras-chave: educação a distância, discriminação, acesso, tecnologia.

\section{Introducción}

Costa Rica, país libre y democrático, ha adoptado por los derechos humanos como un pilar en su gestión, sin distinción de sexo, raza, idioma, religión o cualquier otro aspecto; estos incluyen, entre otras cosas, el acceso a una educación, la salud y una vivienda digna. En esta nación, desde la formación más temprana, se enseña a la ciudadanía que tiene derechos que deben ser respetados y garantizados por el Estado. 
En relación con el ámbito educativo, debido a la pandemia por Covid-19, el Ministerio de Educación Pública de Costa Rica (MEP) realizó el cierre de las clases presenciales, las cuales fueron sustituidas por la modalidad de educación a distancia. Lo anterior llevó a docentes, así como al alumnado, a enfrentarse a un gran cambio en la rutina académica en donde una de las transformaciones más drásticas fueron las clases virtuales por medio de plataformas como Teams.

Lo anterior genera una gran incertidumbre en cuanto a cómo ha sido la educación para el estudiantado durante estos meses. Ello, sobre todo, considerando que el implementar una modalidad a distancia podría perjudicar al estudiantado que no cuenta con acceso a Internet, o bien que no posee los implementos electrónicos para acceder a plataformas como WhatsApp y Teams, lo cual les permitiría mantener una interacción docente-estudiante óptima y asistir a las clases virtuales de forma regular.

Además de lo dicho, debe considerase que la educación a distancia conlleva no solo disponer de Internet e implementos tecnológicos básicos como teléfono celular y computadora, sino, además, poseer las destrezas fundamentales y habilidades básicas en el uso de herramientas tecnológicas. De igual manera, es indispensable que el alumnado posea los conocimientos básicos en acceso a información digital fiable y segura, con los cuales puedan discernir entre datos falsos y reales.

Según lo anotado, la modalidad de educación a distancia podría estar colocando a un sector de la población estudiantil en desventaja, en relación con quienes sí tienen la posibilidad de llevar una formación virtual, con lo que se viola, así, su derecho a una instrucción de calidad y en igualdad de condiciones. Esto puede ocasionar una deserción por parte de estudiantes que se sienten aislados del sistema educativo, o bien la repitencia de grado. A raíz de estas posibles problemáticas, se considera como objetivo del presente estudio "analizar el acceso a la educación de estudiantes de I y II ciclos de la educación general básica, por medio de la modalidad a distancia, durante la pandemia por Covid-19, según el marco de los derechos humanos".

En cuanto a la metodología utilizada, el estudio responde al modelo descriptivo con enfoque cualitativo, cuyo fin es efectuar una explicación de los fenómenos, situaciones, contextos y sucesos estudiados, con lo que se pueda detallar, analizar y hacer una medición respecto a las variables o conceptos, sin llegar a relacionarlos (Hernández et al., 2014). La intención de la investigación descriptiva cualitativa es precisar las propiedades del fenómeno, a través del examen de los resultados obtenidos, con el propósito de realizar una exposición respecto a lo que está siendo indagado (Díaz, 2009). 
Se agrega a lo antes señalado que este es un trabajo de tipo exploratorio no experimental, en el cual no se construye ninguna situación, sino que se observan escenarios ya existentes; en este caso, las variables ya han ocurrido y no pueden ser manipuladas. Además, es importante mencionar que el presente estudio se sustenta en la recolección y análisis de información bibliográfica, por lo que versa sobre aquellas pesquisas previas sobre la calidad de la educación y el acceso a ella, de los estudiantes de I y II ciclos de la educación general básica, en la modalidad a distancia, durante la epidemia por Covid-19 en Costa Rica.

\section{Educación, un derecho para todos y todas}

Costa Rica se caracteriza por poseer una política educativa que fomenta el acceso a la educación de todos y todas por igual. Según el artículo 78 de la Constitución Política, todo niño niña o adolescente que habite en la República tiene el derecho y la obligación de acceder a la educación preescolar, general básica y diversificada. En el mismo sentido, señala dicho ordinal que el Estado facilitará el acceso tecnológico a todos los niveles de la educación. Este derecho es irrestricto, no se puede limitar, por ningún motivo (MEP, 2013).

A su vez, el derecho educativo se basa en la normativa internacional de la Declaración Universal de Derechos Humanos, artículo 26, así como en la Convención sobre los Derechos del Niño (ratificada en Costa Rica mediante la Ley 7184), normas de acuerdo con las cuales todo niño y niña merece una educación gratuita, de la cual dispongan y a la que puedan acceder, con garantía de igualdad de oportunidades para todas las personas (MEP, 2013).

Sin embargo, pese a lo anterior, no existe al momento una entidad encargada de garantizar el acceso a la educación en la modalidad a distancia, la cual contemple las condiciones particulares de cada estudiante, como la disponibilidad de Internet o herramientas tecnológicas para que los alumnos y las alumnas puedan conectarse a clases mediante Teams o mantener contacto con docentes por medio de redes sociales como WhatsApp. Tómese en cuenta que no se midió la habilidad del estudiantado en el manejo de instrumentos tecnológicos y en el acceso a fuentes fiables de información digital.

\section{Importancia de la tecnología en la educación a distancia}

Dada la realidad que se vive en el presente, es fundamental que el modelo educativo se adapte al contexto, donde se procure la búsqueda de recursos que mediaticen la educación actual. Respecto a ello, Porlán (2020) resalta que, en la última década, las estrategias utilizadas por los docentes y las docentes se han dirigido, 
sobremanera, hacia el apoyo de instrumentos que incluyan el uso de tecnologías de la información y la comunicación (TIC), con el objetivo de llevar a cabo el proceso de enseñanza y aprendizaje de una forma más atractiva para el estudiantado. Ante ello, la importancia de provocar una revolución digital, con miras a poner la educación a la altura de estos tiempos.

Respecto al uso de estrategias basadas en la introducción de recursos tecnológicos en la educación, Bailey, Peralta y Aparicio (2020) se fundamentan en una sinergia establecida entre la integralidad y la autonomía del aprendizaje:

la educación a distancia con el uso de las TIC's se caracteriza por tener como objetivo fundamental la formación integral del estudiante a partir del desarrollo de su independencia y su autorregulación, con una concepción del proceso de enseñanza-aprendizaje desarrollador, sin dejar de lado los esfuerzos que los docentes realizan en su afán de proponer nuevas opciones didácticas a sus alumnos (p. 100).

En nuestro país, se han realizado propuestas interinstitucionales entre organizaciones como el IMAS y el MEP, para crear tácticas que garanticen el acceso a la educación de estudiantes que pertenezcan a núcleos familiares en categoría de pobreza o pobreza extrema, en las cuales, por la adquisición de un paquete de Internet económico (3 megas por la suma de 3700 colones), se les otorga una computadora. Pese a esto, no se contempló al alumnado de escasos recursos quienes viven en zonas rurales sin acceso a la red.

\section{Viabilidad de la educación a distancia}

Al hablar de viabilidad en general, se hace referencia a la posibilidad de que algo ocurra, o bien a los medios que se tienen para conseguir un objetivo (Real Academia Española, 2019, definición 3). Se comprende aquí como viabilidad de la educación virtual, entonces, todos aquellos medios y recursos a los que tiene acceso el MEP para garantizar el desarrollo de un proceso de enseñanza- aprendizaje de calidad dirigido a todo el estudiantado por igual, sin discriminación.

Noah (2018) menciona que la educación hoy debería abordar cuatro conocimientos básicos, los cuales califica como las "cuatro ces": pensamiento crítico, comunicación, colaboración y creatividad, todos enmarcados en el desarrollo de la capacidad de adaptarse al cambio. Es decir, se alude a conocer y aprender a utilizar, de forma óptima, los recursos con que se cuenta, de manera que estos potencien nuestro aprendizaje. En este sentido, lo importante es mediar nuevos aprendizajes de la mano del avance tecnológico y científico, mediante los que se logre proporcionar la equidad en el acceso a la tecnología, sobre todo para quienes no se han familiarizado con los avances digitales (Noah, 2018). 
Por otra parte, Parra (2003) establece que uno de los aspectos fundamentales a la hora de fomentar una espiral de conocimiento es la "interacción de la sociedad con el ambiente externo", lo cual se traduce en que las personas logren enfrentarse positivamente a los cambios en su rutina, hábitos o marcos de referencia cognoscitivos. Para lograrlo, es necesario que cada persona consiga cuestionar sus paradigmas y concepciones. Ello quiere incitar a que el crecimiento o la adquisición de nuevos conocimientos se base en la continua adaptación de la sociedad a los cambios que enfrenta, entre estos, los de carácter tecnológico (Parra, 2003).

Entre las ventajas más destacadas de la educación a distancia se encuentra la de colocar la persona estudiante como protagonista de su propio aprendizaje, de modo que el docente o la docente pueda mediar las clases de forma asincrónica y así se estimule el aprendizaje autónomo e integral, al igual que se favorezca la autorregulación. Bajo este paradigma de aprendizaje, se destaca tal formación a distancia como una respuesta para ampliar la cobertura y superar las barreras de espacio y tiempo (Torres, 2017).

De igual manera, es importante considerar que la educación a distancia no conlleva únicamente la virtual, sino que contempla también estrategias como el material impreso, entre otros. En cuanto a esto, Bailey, Peralta y Aparicio (2020) hablan sobre cómo la instrucción a distancia puede entenderse como aquel tipo de educación que se da fuera de la presencialidad e involucra utilizar diferentes medios, tanto de manera sincrónica como asincrónica.

En relación con el párrafo previo, las estrategias o actividades asincrónicas son aquellas que se dan fuera del tiempo sincrónico de una clase. En el caso de este apartado, se contemplan las que, a la par de ser asincrónicas, no implican el tener acceso a Internet. Algunas de ellas son esquemas, planteamiento de problemas cotidianos, actividades de autoevaluación, mapas conceptuales, análisis de casos, resúmenes, líneas de tiempo, analogías o comparaciones, etc. (Andrés et al., 2020).

Cabe mencionar que, a diferencia de cómo se haría en una clase sincrónica, en las lecciones asincrónicas, las estrategias y actividades deben organizarse en un material escrito, el cual debe estar conformado de manera tal que los estudiantes y las estudiantes comprendan el contenido, puedan ponerlo en práctica y alcancen un proceso de autoevaluación, dado que no tienen un contacto diario presencial o virtual con la persona docente.

El Ministerio de Educación Pública costarricense propone la planificación y elaboración de guías de trabajo autónomo, las cuales "deben ser construidas poniendo en práctica múltiples formas de motivación, representación (con materiales diversos 
y accesibles) y expresión. Se debe tener muy presente los diferentes niveles de logro de cada una de las personas estudiantes" (MEP, 2020, p. 17).

Desde el punto anterior y para tener la perspectiva sobre los recursos utilizados por los educadores y las educadoras en tiempos de educación a distancia, se rescatan las herramientas que, de acuerdo con Mendolia (2020), son las más utilizadas en la educación costarricense.

- De comunicación: se consideran los recursos como Facebook, Twitter, WhatsApp y correo electrónico.

- De trabajo académico: se consideran los recursos como Moodle, Google Suite, Google Classroom y Teams.

- De almacenamiento: se consideran los recursos como Dropbox y Google Drive.

- De trabajo sincrónico: se consideran los recursos como Skype, Google Hangouts y Zoom. (p. 10)

Además, en cuanto las desventajas de la formación a distancia, Chaves (2017) menciona, en su estudio sobre la educación a distancia como respuesta a necesidades del siglo XXI, que estas apuntalan el trecho existente entre docente y estudiantado, la mediación pedagógica y la realimentación más lenta. Por ello, esta modalidad puede representar una baja interacción social entre educandos, así como entre estos y el estudiantado.

A modo de corolario, se puede afirmar que hoy la tecnología permite que sea posible implementar un modelo de educación a distancia de calidad. Es importante considerar que los recursos utilizados deben ser garantes del acceso a una óptima formación, con igualdad y sin discriminación para todos los estudiantes y las estudiantes: las estrategias y materiales educativos utilizados en un sistema de educación a distancia deben mediar entre los contenidos y los estudiantes, según los recursos que se tengan disponibles (Andrés et al., 2020).

Como se observa, la educación a distancia, en sí, no es el problema, sino su aplicación en una sociedad que no está aún preparada o capacitada para sobrellevarla. Se desea recalcar que la formación virtual es una opción novedosa, la cual puede ser de mucho provecho para el alumnado. Sin embargo, a la hora de aplicarla en nuestro país, primero se debe garantizar que absolutamente todos los estudiantes y las estudiantes no solo tengan acceso a las herramientas necesarias, sino también a los conocimientos, para que les sea posible hacer un uso idóneo de estos. 


\section{Educación a distancia en Costa Rica}

En el 2020, el Ministerio de Educación Pública de Costa Rica implementa la modalidad de educación a distancia como medida para frenar el contagio por Covid-19, lo cual viene a marcar un hito en la educación costarricense, ya que se suspendieron las clases presenciales y en su lugar se implementó la modalidad educativa virtual, por medio de plataformas como Microsoft Teams y WhatsApp.

Este súbito paso de la presencialidad a la digitalización educativa representó para el estudiantado no solo un cambio drástico en su rutina académica, sino una presión y demanda imprevista de aquellos recursos indispensables cuando se accede a esta modalidad de educación: un equipo electrónico (como computadora o, en su defecto, un teléfono celular), además de una conexión a Internet de velocidad media como mínimo.

Aquí es importante considerar que Costa Rica es un país con gran cantidad de zonas rurales periféricas, en las cuales la población no cuenta con acceso a Internet de calidad, pues no existen ni siquiera empresas que lo distribuyan. Asimismo, se encuentra otro sector poblacional que sí cuenta con acceso a la red e implementos electrónicos, pero que no posee los conocimientos ideales para utilizar eficazmente plataformas como las antes descritas, o bien el acceso al correo institucional, el medio oficial de comunicación entre el centro educativo y el estudiantado.

Aunado a ello, se debe contemplar que un paquete de Internet de velocidad media (entre 6 y 15 megas) tiene un precio que ronda los 15000 colones mensuales, monto que se sale del presupuesto de muchas familias en condición de pobreza y pobreza extrema. Este problema se refleja en un estudio del Estado de la Educación, en el cual se indica que un $17 \%$ de el estudiantado evidenció no tener acceso a un Internet estable, y en el que se dice, además, que un $3 \%$ no poseía Internet del todo (León y Gómez, 2020). De igual forma, en el caso personal docente, el trabajo aludido señala que se presentaron este tipo de inconvenientes, pues un $25 \%$ del personal comunicó tener conexiones inestables o no contar, del todo, con acceso a Internet; debió trabajar con el sistema de copias que planteó el MEP, en el cual no existe ningún tipo de mediación docente-alumnado. En razón de este contexto, es importante considerar que ese $25 \%$ de docentes laboran en instituciones públicas pertenecientes a zonas como Sulá, Peninsular, Grande de Térraba, Aguirre, Santa Cruz, Nicoya, Coto y Cañas, entre las principales (León y Gómez, 2020).

También es fundamental tener presente que por cada docente sin acceso a Internet hay más de 10 estudiantes a quienes no se les garantiza el derecho a la educación en igualdad de condiciones que al resto de los niños y las niñas del país, lo cual 
podría representar, a largo plazo, un terrible rezago formativo. Según el Estado de la Nación, hay cerca de 2397 profesores que, para mediados de año, aún no habían logrado establecer contacto con sus estudiantes (León y Gómez, 2020). Este contexto deja entender que podría tratarse de más de 548300 estudiantes sin acceso a educación. Lo más preocupante del caso, es que dicho fenómeno es propio de la educación primaria en las regiones rurales de Alajuela, Cartago, San Carlos y Limón. Ello deja ver que no se trata solo de una complicación de acceso a Internet, sino del poco apoyo que reciben el personal docente y educandos en estas condiciones (León y Gómez, 2020).

Se trata, en resumen, de la exclusión y discriminación de una parte importante de la población estudiantil, quienes no contaban con los recursos ni conocimientos (alfabetización digital) para acceder a este tipo de modalidad educativa. Esto trae serias repercusiones, puesto que el derecho a la educación estaría siendo violentado para toda población estudiantil; su desarrollo formativo, integral y académico se ve comprometido, haciendo aún más profundas las ya existentes brechas en el sistema educativo.

En relación con la postura del MEP, este afirma que, desde una educación a distancia, "Las orientaciones aquí dispuestas se crearon tomando en consideración las necesidades y contextos de la población estudiantil; sin embargo, existe la posibilidad de que deban ajustarse sobre la marcha en el tiempo, dependiendo de las indicaciones de las autoridades" (MEP, 2020b, p. 6).

Según las cuestiones señaladas por el MEP (2020b), sobre las orientaciones para el soporte del proceso educativo a distancia, el caso de la población estudiantil que no posee Internet implica que el docente o la docente es responsable de buscar las maneras y medios para que se haga una adaptación de material didáctico, utilizar herramientas que le permitan elaborar el instrumental pertinente y movilizar el contacto con el estudiantado, apoyado en su base familiar.

Por tanto, la preocupación por la enseñanza desde la modalidad a distancia y las implicaciones que el profesorado ha tenido que tomar en cuenta para responder al estudiantado que no cuenta con acceso a Internet en sus hogares, plantea una inquietud fundamentada en la educación desde este escenario. Lo óptimo sería que toda persona estudiante pueda gozar de la oportunidad de tener una red fija en su domicilio, pero el contexto dista de esa realidad y se representa, así, un obstáculo para ofrecer una educación de calidad, que favorezca el desarrollo de habilidades y competencias durante este ciclo lectivo y los venideros. 


\section{Principales herramientas tecnológicas utilizadas}

El cambio en el modelo educativo enfrentó al estudiantado a herramientas tecnológicas que no conocían y para las cuales requerían disponer de destrezas básicas que les permitieran acceder a estas de forma efectiva. Esto vislumbró que existe una significativa brecha tecnológica entre la población estudiantil de la educación general básica: un gran porcentaje de los alumnos señaló que no contaban con formación en herramientas virtuales educativas, del cual su mayoría utilizó la plataforma WhatsApp como la única vía de comunicación con los docentes y las docentes (León y Gómez, 2020).

Cabe recalcar que, en el caso del profesorado, aun cuando sí se tiene acceso a Internet, tampoco se asegura que pueda garantizar el derecho a la educación de sus alumnos y alumnas, pues la mayor parte del gremio docente no posee formación en herramientas virtuales para la enseñanza, ni en la universidad ni a lo largo del ejercicio de su carrera profesional. Lo dicho se refleja en las herramientas utilizadas por los maestros y las maestras durante la pandemia por Covid-19 (ver gráfico 1): los videos, el material impreso y la comunicación por WhatsApp fueron los medios más empleados (León y Gómez, 2020).

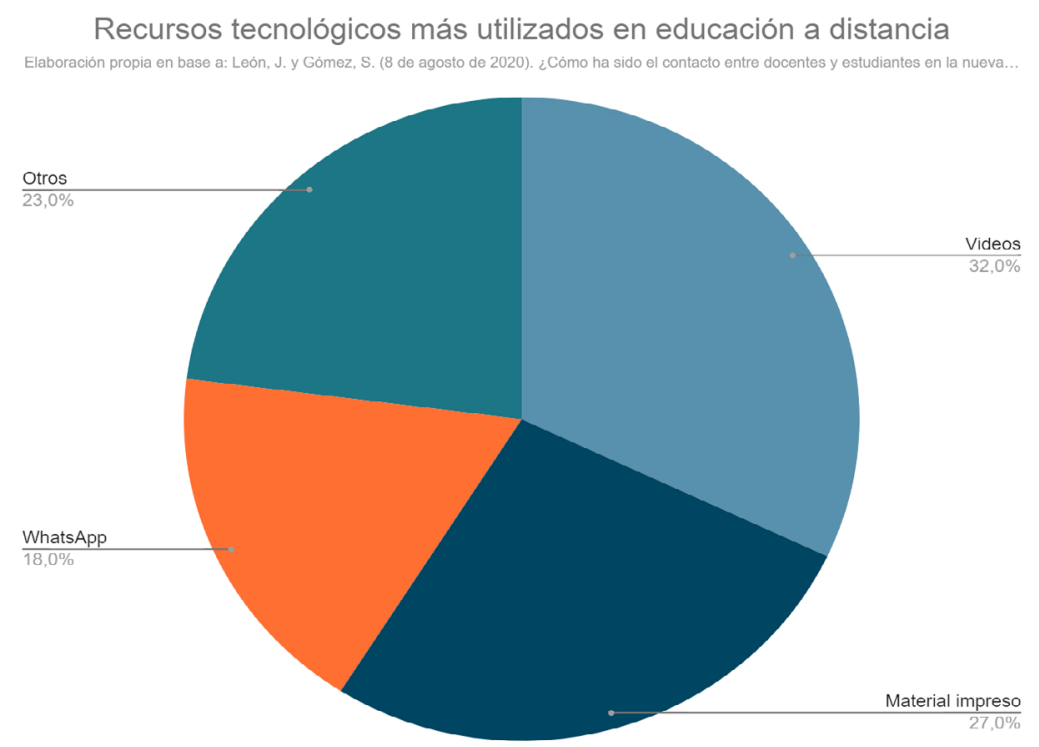

Pese a que existe una gran disponibilidad de recursos tecnológicos óptimos para ser utilizados en educación, la realidad costarricense es que se sigue haciendo 
uso de los más básicos. Esto, en buena medida, porque la mayoría de docentes y estudiantes no cuenta con el conocimiento y las habilidades tecnológicas para emplear otras herramientas, tales como plataformas educativas, entre las cuales se destaca Claroline y Kahoot; plataformas on line como Kanva, Gennialy y Actívate; aplicaciones educativas como Tinkercad; entre otros.

\section{Conclusiones}

En cuanto a la disponibilidad de recursos tecnológicos, sí existen actualmente herramientas y medios para garantizar el derecho a la educación, ya que, como se mencionó, se cubren niveles de comunicación, almacenamiento, trabajo académico y sincrónico. A la par de esto, se hallan recursos gratuitos que pueden ser utilizados en el nivel académico, generando componentes de gran calidad, como Gennialy y Canva. También, hay disponibles juegos interactivos que pueden modificarse de acuerdo con los temas abordados en las diferentes materias.

Sin embargo, entre las aplicaciones usadas en nuestro país, la más frecuente en la educación a distancia fue la plataforma WhatsApp, la cual resulta vital en el proceso de formación virtual realizado desde el 2020. Esta herramienta fue la más utilizada por estudiantes que no contaban con computadora ni Internet fijo; resultó el principal medio de las personas docentes para hacer llegar videoclases, audios, material extra y llamadas que cumplían funciones de evaluación, así como de acompañamiento académico y emocional.

Aunado a lo anterior, el personal docente, sobre todo de primaria, recurrió a material impreso, con el objetivo de alcanzar a que no contaban con Internet o equipo tecnológico. En este punto, aunque es importante resaltar el compromiso docente por buscar métodos alternativos para desarrollar las diferentes estrategias, se hace indispensable señalar que dichos medios resultan rígidos y con poco espacio para la participación activa del estudiantado, lo cual coloca a este último en una posición de desventaja frente a los recursos tecnológicos, tanto como denuncia una discriminación en relación con aquellos que sí pueden comunicarse a diario con sus docentes.

Si bien el presente curso lectivo fue atípico y exigió al personal docente salir de su zona de confort, para diseñar nuevas estrategias que les permitieran cumplir con los objetivos de clase, a la par se debe considerar que la gran mayoría logró llegar al alumnado, sea mediante recursos virtuales o por medio de material impreso. La verdad, eso sí, es que se evidenció una brecha tecnológica importante entre los estudiantes de zonas rurales y urbanas, al igual que de pobreza y pobreza 
extrema respecto de sectores medios y altos, la cual se replica, incluso, entre las personas docentes.

El panorama es preocupante, no solo porque se está recargando al educador y a la educadora una responsabilidad que no le compete, pues es deber directo del Ministerio de Educación Pública velar porque el estudiantado tenga acceso a una educación de calidad, sino, además, porque los derechos humanos contemplan el acceso a una formación óptima y en igualdad de condiciones para todos. Diferenciar las estrategias de mediación utilizadas entres quienes sí tienen acceso a la educación virtual y los que no, violenta el derecho educativo de la población más vulnerable.

Considerando lo dicho, es necesario concluir que dado el problema en cuanto al acceso a recursos tecnológicos por parte de estudiantes, el personal docente busca garantizar la formación por otros medios como el material impreso. Este no iguala la calidad de la educación que recibieron los alumnos y las alumnas quienes disponían de medios tecnológicos, los cuales sí permiten una comunicación constante con el profesorado, a la par del acceso a herramientas complementarias que fomentan una mejor comprensión de cada tema.

La situación descrita implica que en Costa Rica se ha violentado y sigue en riesgo el derecho a la educación, sobre todo, en aquellos casos en los que un apersona estudiante no tiene acceso a dispositivos electrónicos, ni siquiera a un teléfono celular para hacer llamadas (la única comunicación con el docente es el material impreso, como ya se refirió). Dado que tenemos conciencia de que esta es la realidad de muchos niños y niñas en el país, toca ahora visualizar acciones urgentes que posibiliten restituir, compensar y garantizar su derecho a la formación.

\section{Recomendaciones}

Por todo lo anterior, se recomienda, en primer lugar, que el Estado, mediante entidades de ayuda social y otras organizaciones, garantice el acceso a la red de todo el estudiantado, sea a través de la dotación de equipos de cómputo y paquetes de Internet o, en su defecto, con laboratorios de cómputo comunales, que estén a disposición de aquellos alumnos quienes residen en lugares donde no hay acceso a Internet $o$ este es limitado.

También, se sugiere que el MEP brinde talleres de capacitación para docentes y estudiantes, en general, sobre el uso de implementos tecnológicos y herramientas académicas, con el fin de que todas las personas involucradas en la educación a distancia aprovechen al máximo los recursos gratuitos disponibles hoy, en pro de mejorar el ambiente virtual educativo. 
Además, es indispensable que se realice una evaluación profunda de la viabilidad de la educación a distancia, con el fin de llevar a cabo las mejoras requeridas, para que a futuro se convierta en un modelo formativo de calidad, paralelo a las clases presenciales. Para esto, es necesario que el MEP evalúe, modifique y perfeccione las condiciones actuales, de forma que se garantice el acceso a una educación óptima y en igualdad de condiciones para todo el estudiantado.

\section{Referencias}

Andrés, C., Anchetta, G., Barbozas, Y. y Peraza, M. (2020). Estrategias de mediación pedagógica de las unidades didácticas de la Universidad Estatal a Distancia de Costa Rica. UNED Research Journal, 12(1), 18-56. https://revistas.uned.ac.cr/index.php/ cuadernos/article/view/2940/3654

Bailey, L., de Peralta, M. y Aparicio, J. (2020). Teoría y prácticas de aprendizaje de la educación a distancia. Guacamaya, 5(1), 97-108. https://revistas.up.ac.pa/index.php/ guacamaya/article/view/1784/1354

Chaves Torres, A. (2017). La educación a distancia como respuesta a las necesidades educativas del siglo XXI. Revista Academia y Virtualidad, 10(1), 23-41.

León, J. y Gómez, S. (8 de agosto de 2020). ¿Cómo ha sido el contacto entre docentes y estudiantes en la nueva modalidad de clases a distancia? Programa de Estado de la Nación. https://estadonacion.or.cr/como-ha-sido-el-contacto-entre-docentes-y-estudiantes-en-la-nueva-modalidad-de-clases-a-distancia/

Mendiola, M., Hernández, A., Torres, R., Carrasco, M., Romo, A., Mario, A. y Cazales, V. (2020). Retos educativos durante la pandemia de COVID-19: una encuesta a profesores de la UNAM. Revista Digital Universitaria, 21(3), 1-67.

Ministerio de Educación Pública. (2013). La educación, un derecho de todos y todas: Compendio de normas acerca del derecho a la Educación de la población migrante y refugiada. San José, Costa Rica.

Ministerio de Educación Pública. (2020b). Orientaciones para el apoyo del proceso educativo a distancia. https://www.mep.go.cr/sites/default/files/orientaciones-proceso-educativo-distancia_0.pdf

Noah, Y. (2018). 21 lecciones para el siglo XXI. (6. ${ }^{\mathrm{a}}$ ed.). 47-49. 08021 Barcelona. https:// pmadsena.weebly.com/uploads/1/2/7/1/12712314/21_lecciones_para_el_siglo_xxi_-yuval_n.pdf

Parra, I. (2003). Los modernos alquimistas. Epistemología corporativa y gestión del conocimiento. (1. ${ }^{\mathrm{a}}$ ed.). 7-50. https://repository.eafit.edu.co/bitstream/handle/10784/71/9588173736.pdf? sequence=1\&isAllowed=y 
Porlán, R. (2020). El cambio de la enseñanza y el aprendizaje en tiempos de pandemia. Revista de Educación Ambiental y Sostenibilidad, 3 (1), 74-103 1502-1502.

Real Academia Española. (2019). Diccionario de la lengua española. (23. a ed.). https:// dle.rae.es/posibilidad 